\title{
Polarization-dependent Rabi oscillations in single InGaAs quantum dots
}

\author{
L Besombes ${ }^{1}$, J J Baumberg ${ }^{1,2}$ and J Motohisa ${ }^{2}$ \\ ${ }^{1}$ Department of Physics and Astronomy, University of Southampton, \\ Southampton SO17 1BJ, UK \\ ${ }^{2}$ Research Center for Integrated Quantum Electronics, Hokkaido University, \\ Sapporo 060-8628, Japan
}

Received 28 July 2003

Published 3 March 2004

Online at stacks.iop.org/SST/19/S148 (DOI: 10.1088/0268-1242/19/4/052)

\begin{abstract}
Measurements of optical Rabi oscillations in the excited states of individual InGaAs are presented. Under pulsed resonant excitation we observe Rabi oscillations with increasing pulse area, which are damped after the first maximum and minimum. We show that the observed damping comes from an additional non-resonant generation of carriers in the quantum dot. The observation of Rabi oscillations provides an efficient way of directly measuring the excitonic transitions' dipole moments. A polarization anisotropy of the dipole moment is resolved in some of the quantum dots.
\end{abstract}

\section{Introduction}

Since the development of local spectroscopy techniques, the atomic-like optical properties of semiconductor quantum dots (QDs) have been intensively studied. Discrete electronic states of single QDs can be considered as localized two-level systems. The increased interest in QDs has been related to the potential use of these two-level systems as building blocks of prospective quantum logic gates $[1,2]$. The development of techniques to perform coherent manipulation of carrier wavefunctions in single QDs is a key point for a successful implementation of quantum information processing in these solid-state systems.

Optical Rabi oscillations are examples of non-linear coherent light-matter interactions, which may provide coherent control in solid-state devices [3, 4]. When a twolevel system is exposed to a strong light field, the inversion of this system oscillates at the Rabi frequency, which is proportional to the dipole moment of the transition $\mu$ and the optical electric field $\epsilon(t)$. Under pulsed resonant excitation, the number of complete oscillations depends on the area of the light pulses $\theta(t)=2 \mu / \hbar \int_{-\infty}^{t} \epsilon\left(t^{\prime}\right) \mathrm{d} t^{\prime}$ and the Rabi oscillations manifest themselves as a sinusoidal dependence of the population inversion on the pulse area [5]: the time integrated Rabi oscillations.

In this work we present measurements of optical Rabi oscillations in the excited states of individual InGaAs QDs under pulsed resonant excitation. We observe Rabi oscillations, which are damped versus pulse area after the first maximum and minimum. We show that the observed damping comes from the additional non-resonant generation of carriers in the QD due to indirect transitions involving confined states of the QD and delocalized states of the wetting layer. Sensitive determination of the dipole moments obtained from polarization measurements reveals an anisotropy in some of the QDs.

\section{Experimental details}

The investigated self-assembled QDs are grown on GaAs substrates using metal-organic vapour phase epitaxy. The deposition of a reduced amount of InAs, 1.8 ML (close to the critical thickness of the dot formation in our growth condition) is employed to produce a sparse QD density of about 1-5 $\times$ $10^{9} \mathrm{~cm}^{-2}$. The low temperature $(T=5 \mathrm{~K})$ photoluminescence (PL) of individual QDs is excited and collected through large numerical aperture microscope objectives and shadow masks with $0.2-1.0 \mu \mathrm{m}$ apertures. For the photoluminescence excitation spectroscopy (PLE), QDs are excited with a tunable Ti:sapphire laser and a cooled CCD camera is used for parallel detection of the complete PL of the s-shell (exciton ground state emission region) as a function of the laser excitation energy. 


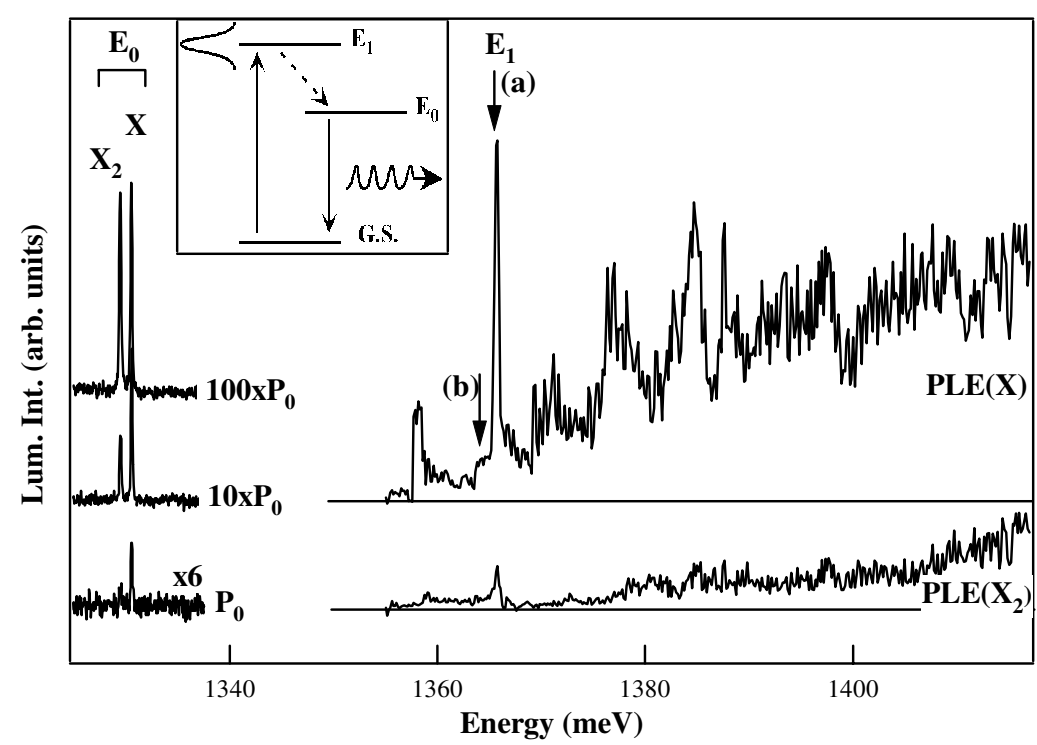

Figure 1. PL and PLE spectra of an individual QD. PL spectra are obtained under non-resonant excitation at $\lambda_{\text {exc }}=532 \mathrm{~nm}$. Arrows indicate the wavelengths of excitation for the power dependence presented in figure 2. Inset: energy diagram of the optical transitions discussed in the text.

\section{Results and discussion}

PL and PLE spectra from a typical individual QD are presented in figure 1. At low excitation densities, a single narrow line (labelled X) dominates the PL spectra. For higher powers, a second emission line $\left(\mathrm{X}_{2}\right)$ appears on the low-energy side of $\mathrm{X}$. The linear and the quadratic power dependences of $\mathrm{X}$ and $\mathrm{X}_{2}$ respectively identify these lines as the recombination of the exciton and biexciton in the same QD. The X PLE spectrum presents different sharp absorption lines corresponding to confined excitonic transitions, and a continuum absorption background progressively increasing until the threshold of the wetting layer. It has been recently shown (see [4]) that this continuum of absorption is related to the generation of carriers in the QD by spatially indirect transitions involving the fundamental state $E_{0}$ of the QD and delocalized interface states of the wetting layer. The strength of this background depends on the local environment of the QD and is different from dot to dot.

As illustrated in the inset of figure 1, if the absorption background is ignored, a QD under resonant excitation can be considered as a discrete three-level system. Since in these QDs the relaxation time from the p-state $E_{1}$ to the s-state $E_{0}$ is shorter than any recombination time [7], the PL intensity of the fundamental state $E_{0}$ can be used to monitor the population of the resonantly excited excitonic transition $E_{1}$ as a function of the pulsed excitation density [8].

The evolution of the intensity of the complete PL of the s-shell is presented in figure 2 for two different excitation wavelengths as a function of the square root of the pulsed excitation intensity (a quantity proportional to the integrated area of the 3 ps Gaussian pulses). Under resonant excitation on the first excited state $E_{1}$ (figure $2(a)$ ), with increasing excitation intensity $I_{\text {res }}$ we first observe an increasing PL from $X$. Then the PL reaches a maximum; a further increase of $I_{\text {res }}$ then leads to a reduction of the PL. Simultaneously, the PL intensity of the biexciton increases slowly. The oscillation of

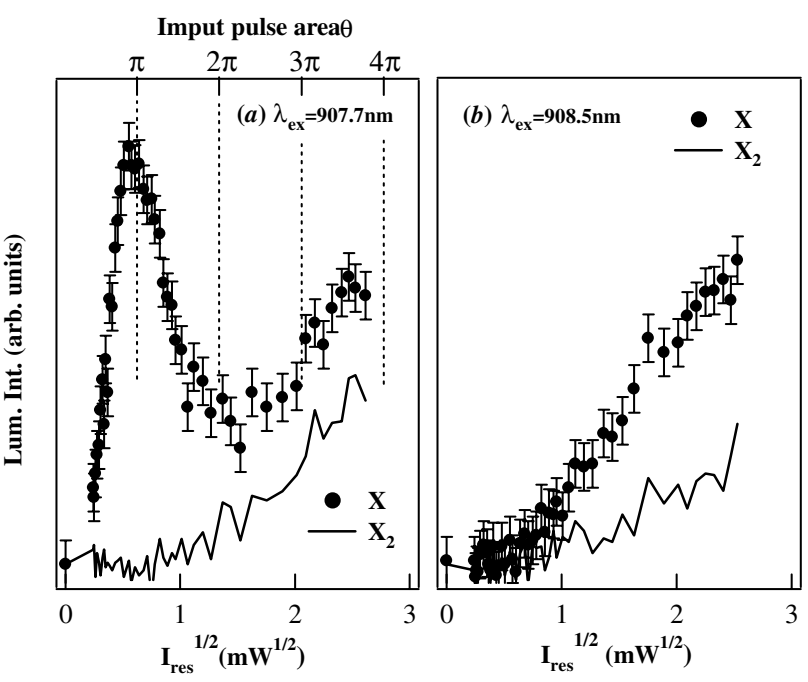

Figure 2. Evolution of the $\mathrm{X}$ and $\mathrm{X}_{2} \mathrm{PL}$ intensity under circularly polarized pulsed resonant excitation on $E_{1}(a)$ and under non-resonant excitation at $\lambda_{\mathrm{exc}}=908.5 \mathrm{~nm}(b)$.

the X PL intensity occurs when the QD is only occupied by a single exciton as almost no biexciton contribution is observed in this excitation regime. This saturation effect cannot be due to the formation of multi-exciton complexes as usually observed under non-resonant excitation. The oscillation of $\mathrm{X}$ is a direct manifestation of Rabi oscillations in the resonantly excited state $E_{1}$ which behaves as a discrete atomic level. The population of $E_{1}$ is maximum for an optical $\pi$ pulse, decreasing as the excitation intensity is further increased and reaching a minimum for a $2 \pi$ pulse. In this three-level system, a portion of the excited state population can lose its phase coherence or relax to $E_{0}$ during the 3 ps excitation pulses and contribute to the residual PL observed for a $2 \pi$ pulse. However, the observed Rabi oscillations appear strongly damped at high $I_{\text {res. }}$ Even with the fast dephasing induced by the population relaxation from $E_{1}$ to the fundamental state $E_{0}$, in a pure 

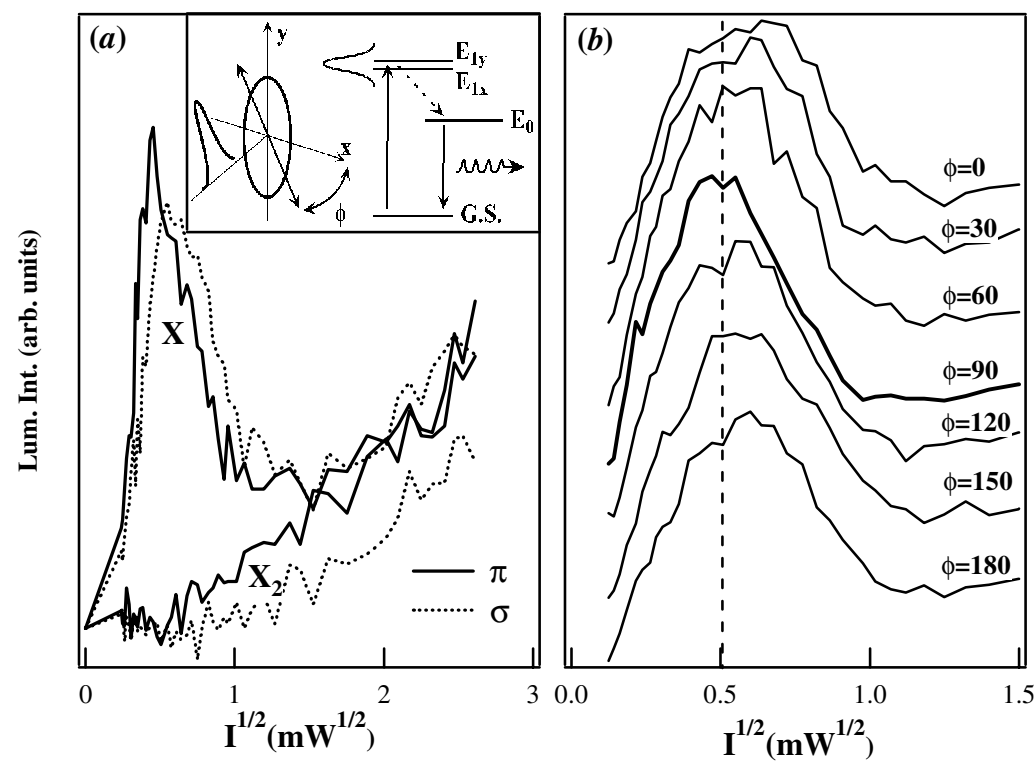

Figure 3. (a) Comparison of the $\mathrm{X}$ and $\mathrm{X}_{2}$ PL power dependence obtained under circularly polarized $(\sigma)$ and linearly polarized $(\pi)$ pulsed resonant excitation. (b) Evolution of the Rabi oscillations as a function of the linear polarization direction of the pulsed resonant excitation (spectra are offset for clarity). The geometry of the experiment is presented in the inset of $(a)$.

three-level system several oscillations should be observed at higher excitation density [9].

The disappearance of the Rabi oscillations occurs in an excitation intensity regime where the contribution of the biexciton becomes predominant. For a pure isolated threelevel system and with spectrally narrow excitation pulses, because of the Coulomb shift induced by an exciton already present in the QD, the absorption of a second exciton and the formation of a biexciton should not be observed under resonant excitation. To understand the origin of the Rabi damping and the origin of the biexciton appearance under pulsed resonant excitation, power-dependent measurements were also performed slightly out of resonance. As illustrated in figure $2(b)$, for an excitation wavelength slightly tuned above the discrete transition $E_{1}$, oscillations are no longer observed in the X PL intensity. However, at high excitation density, the emission of the s-shell obtained under resonant and non-resonant excitation is of the same order of magnitude showing that in this excitation regime the main absorption contribution does not come from the discrete excited state but from the continuum PLE background. The presence of these non-resonantly created carriers in the QD shifts the excited state absorption energy and prevents the observation of the Rabi oscillations at high excitation density. The formation of the biexciton also comes from this additional non-resonant generation of carriers superimposed on the resonant excitation.

Due to the Pauli exclusion principle, the creation of the biexciton is reduced if a spin-polarized population of exciton is created by circularly polarized pulses. This effect is illustrated in figure 3(a) where the power dependences of $\mathrm{X}$ and $\mathrm{X}_{2}$ PL intensity are presented for linearly and circularly pulsed resonant excitation. This implies that the continuum background absorption preserves some of the spin selection rules, and that spin scattering is slower than recombination. A change from linear to circular polarization also produces a shift in the position of the maximum occupancy of the excited state. The position of this maximum, corresponding to an optical $\pi$ pulse, is directly related to the dipole moment of the excited transition through the definition of the pulse area. This experiment then provides an efficient way of estimating the dipole moment of the resonantly excited transitions $[8,10]$.

To analyse in detail the effect of the optical polarization on the Rabi oscillations, power dependences are systematically recorded for different directions of linear polarization. A shift of the maximum of the oscillations is observed when the linear polarization direction is progressively rotated through a $180^{\circ}$ angular range (figure 3(b)). This shift clearly resolves the anisotropy of the dipole moment of the excited state.

An anisotropy of the optical properties of the excited state in elongated QDs has been theoretically predicted [11] and directly observed in larger QDs formed by interface fluctuations in narrow quantum wells [12]. In a slightly anisotropic QD, the twofold degeneracy of the first exited state $E_{1}$ is lifted and the resonance is composed of two optically accessible states, $E_{1 X}$ and $E_{1 Y}$, orthogonally polarized. The directions of linear polarizations correspond to the principal axes of the anisotropic confinement potential. Even if the anisotropy-induced splitting is beyond the spectral resolution of PLE measurement, the linear polarization dependence of the Rabi oscillations directly reveals the anisotropy of the dipole moments. In this experiment we rotate the polarization of the pulse sequence with respect to the sample eigen-axes and generate a wavefunction composed of a superposition of the two eigenstates $\left|E_{1 X}\right\rangle$ and $\left|E_{1 Y}\right\rangle$. The effective dipole moment of the transition is then a linear combination of the dipole moment of the two eigenstates and progressively changes from $\mu_{1 X}$ to $\mu_{1 Y}$ as we rotate the polarization direction. An anisotropy of the dipole moment of almost $20 \%$ is measured with a variation from 8 Debye to 10 Debye when the polarization direction is changed by an angle of $90^{\circ}$ between the two cleaved edges of the sample along [110] and [1 $1 \overline{1} 0]$ axes. However the $E_{0}$ peak PL intensity does not change 
Polarization-dependent Rabi oscillations in single InGaAs quantum dots

as the polarization is rotated, proving that only one electronhole pair can be injected into the QD, due to the resulting Coulomb level shifts.

\section{Conclusion}

In summary, we applied micro-spectroscopy to study the coherent non-linear optical properties of the excited states of individual InGaAs QDs. The Rabi oscillations measured in the excited states under pulsed resonant excitation are strongly damped by the additional non-resonant generation of carriers in the QD. This result shows how the coupling with the surrounding medium is important for the development of any device based on coherent optical control of excitonic transitions in individual QDs. The high sensitivity of these measurements also reveals the anisotropy of the dipole moments in anisotropic QDs.

\section{Acknowledgments}

This work has been supported by the European Union through the Research Project SQID IST-1999-11311. JJB thanks Oliver Wright (Hokkaido University) for his hospitality.

\section{References}

[1] Troiani F, Hohenester U and Molinari E 2000 Phys. Rev. B 62 R2263

[2] Biolatti E, D'Amico I, Zanardi P and Rossi F 2002 Phys. Rev. B 65075306

[3] Stievater T H, Li X, Steel D G, Gammon D, Katzer D S, Park D, Piermarocchi C and Sham L J 2001 Phys. Rev. Lett. 87133603

[4] Zrenner A, Beham E, Stufler S, Findeis F, Bichler M and Abstreiter G 2002 Nature $\mathbf{4 1 8} 612$

[5] Gibbs H M 1969 Phys. Rev. A 73446

[6] Vasanelli A, Feirrera R and Bastard G 2002 Phys. Rev. Lett. 89 216804

[7] Besombes L, Baumberg J J and Motohisa J 2003 Phys. Rev. Lett. 90257402

[8] Htoon H, Takagahara T, Kulik D, Baklenov O, Holmes A L and Shih C K 2002 Phys. Rev. Lett. 88 087401

[9] Borri P, Langbein W, Schneider S, Woggon U, Sellin R L, Ouyang D and Bimberg D 2002 Phys. Rev. B 66 081306

[10] Kamada H, Gotoh H, Temmyo J, Takagahara T and Ando H 2001 Phys. Rev. Lett. 87246401

[11] Takagahara T 2000 Phys. Rev. B 6216840

[12] Bonadeo N H, Erland J, Gammon D, Park D, Katzer D S and Steel D G 1998 Science 2821473 